\title{
CFD Modeling of Thermoelectric Generators in Automotive EGR-coolers
}

\author{
Olle Högblom and Ronnie Andersson
}

\author{
Department of Chemical and Biological Engineering, Division of Chemical Engineering, \\ Chalmers University of Technology, SE-412 96 Gothenburg, Sweden.
}

\begin{abstract}
A large amount of the waste heat in the exhaust gases from diesel engines is removed in the exhaust gas recirculation (EGR) cooler. Introducing a thermoelectric generator (TEG) in an EGR cooler requires a completely new design of the heat exchanger. To accomplish that a model of the TEG-EGR system is required. In this work, a transient 3D CFD model for simulation of gas flow, heat transfer and power generation has been developed. This model allows critical design parameters in the TEG-EGR to be identified and design requirements for the systems to be specified. Besides the prediction of Seebeck, Peltier, Thomson and Joule effects, the simulations also give detailed insight to the temperature gradients in the gas-phase and inside the thermoelectric (TE) elements. The model is a very valuable tool to identify bottlenecks, improve design, select optimal TE materials and operating conditions. The results show that the greatest heat transfer resistance is located in the gas phase and it is critical to reduce this in order to achieve a large temperature difference over the thermoelectric elements without compromising on the maximum allowable pressure drop in the system. Further results from an investigation of the thermoelectric performance during a vehicle test cycle is presented.
\end{abstract}

Keywords: thermoelectricity, heat transfer, CFD, EGR

PACS: 47.11.-j , 47.27.T-, 44.25.+f, 44.27.+g, 44.20.+b, 84.60.Rb, 85.80.-b, 79.10.N-

\section{INTRODUCTION}

Waste heat in the exhaust gases from diesel engines is continuously removed in the exhaust gas recirculation (EGR) cooler. If this energy partially could be converted to useful electric energy using thermoelectric elements it would give a significant environmental and economical impact [1]. One opportunity to implement TEG in automotive systems is by integrating it into the exhaust gas recirculation (EGR) system. The EGR technique is used to lower the emissions (NOx) from internal combustion engines by lowering the combustion temperatures. In the EGR a portion of the hot gases from the combustion engine are cold before recirculated back to the engine cylinders. Since NOx primarily forms at high combustion temperatures significant reduction of nitrogen oxide emissions is achieved. The waste heat in the exhaust gases could be used as source for heat flow through a TEG. The average gas temperature into the TEG would approximately be $360^{\circ} \mathrm{C}$. On the cold side the temperature would be close to $90{ }^{\circ} \mathrm{C}$ utilizing the vehicle coolant system. In this work the implementation of thermoelectric generators (TEG) for waste heat recover in automotive exhaust gas is studied, and the critical design parameters highlighted.

High heat transfer in the gas phase will be required. This can be difficult to achieve so one question is what surface temperature the thermoelectric material will sense. Another what possible designs can be used to retain a low pressure drop in the system and at the same time obtain satisfying temperatures in the thermoelectric material. There is also one question regarding the effects the cycling gas temperatures, mass flow rates, and species composition have on the TEG performance. To answer all these questions a model that accounts for fluid flow, heat transfer and power generation from first principles is needed. Besides the prediction of heat transfer, Seebeck potential, Peltier, Thomson effects and Joule heating, the simulations must also give detailed insight to the temperature gradients in the gas-phase and inside the TE elements. In this work a 3D CFD model that accounts for these phenomenon is developed. Boundary conditions in terms of exhaust gas data from a vehicle test cycle is used to study the effects of transients in the system. 


\section{SIMULATIONS}

In this work a 3D CFD model for gas flow and heat transfer has been used together with an in-house code for predicting the thermoelectric performance during a reduced vehicle test cycle. Since the Reynolds number implies turbulent flow conditions turbulence needs to be modeled for the TEG-EGR system. The continuity equation for incompressible flows reads

$$
\frac{\partial\left\langle U_{i}\right\rangle}{\partial x_{i}}=0
$$

and the Reynolds Averaged Navier-Stokes (RANS) equations

$$
\frac{\partial\left\langle U_{i}\right\rangle}{\partial t}+\left\langle U_{j}\right\rangle \frac{\partial\left\langle U_{i}\right\rangle}{\partial x_{j}}=-\frac{1}{\rho} \frac{\partial}{\partial x_{j}}\left\{\langle P\rangle \delta_{i j}+\mu\left(\frac{\partial\left\langle U_{i}\right\rangle}{\partial x_{j}}+\frac{\partial\left\langle U_{j}\right\rangle}{\partial x_{i}}\right)-\rho\left\langle u_{i} u_{j}\right\rangle\right\} .
$$

In the CFD simulations the SST k- $\omega$ model was used to close the Reynolds stresses, $\rho\left\langle u_{i} u_{j}\right\rangle$, that appears in Eq. 2. This model is robust and the cost of implementing it for simulations of engineering applications is affordable although a dense mesh is required at all gas boundaries [2]. Models for the thermoelectric effects [3] were implemented in the in-house code. After regression analysis of measurement data published recently [4], the characteristic thermoelectric properties were implemented in the model. Any other TE material can easily be used in the simulations.

\section{RESULTS}

The results show that a large heat transfer resistance occurs in the gas phase. Figure 1a shows the temperature distribution in the TEG-EGR system with gas flow parallel to the TE surface, Figure 1b shows the potential distribution in the thermocouple. As shown in Figure 1a there is a significant temperature gradient in the gas phase, the temperature drops from the gas bulk to the TE material. As a consequence the TE surface temperature is approximately $160{ }^{\circ} \mathrm{C}$ lower than the gas bulk temperature. The high thermal conductivity in the copper causes obviously no significant gradient. Only about $2 / 3$ of the total temperature difference occurs over the thermoelectric material, as shown in Table a, for a specific pressure drop. The results show clearly that it is essential to reduce the heat transfer resistance in order to achieve a large temperature difference over the thermoelectric elements.
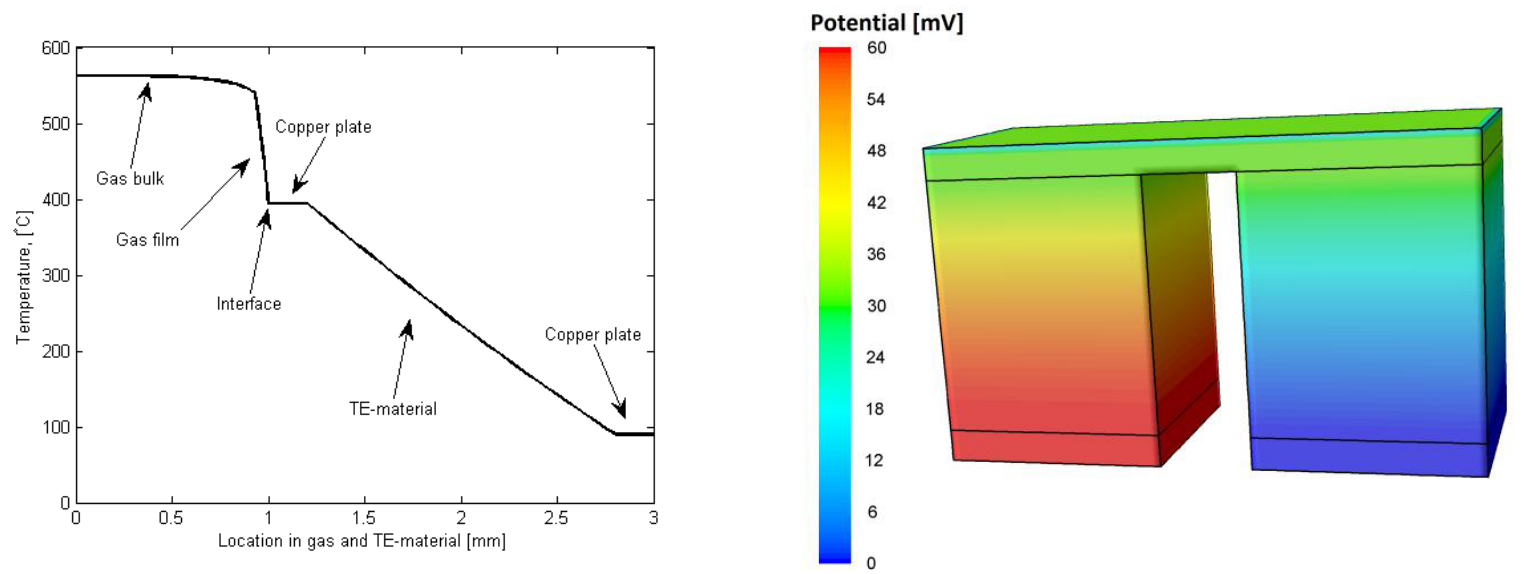

FIGURE 1. (a)Temperature distribution in the TEG-EGR system (b) Contour plot of the total potential.

This problem can be solved by increasing the heat transfer to the surface, but for an EGR application it is of great importance to maintain a low pressure drop in the gas, which to some extent contradicts the requirement of high heat transfer. Hence there is a limitation in just increasing the turbulence to promote higher heat transfer and to increase the surface temperature. When developing new TEG systems it is consequently important to focus on designing high 
heat transfer and not only on developing the TE materials. Further studies is needed to evaluate new low pressure drop designs.
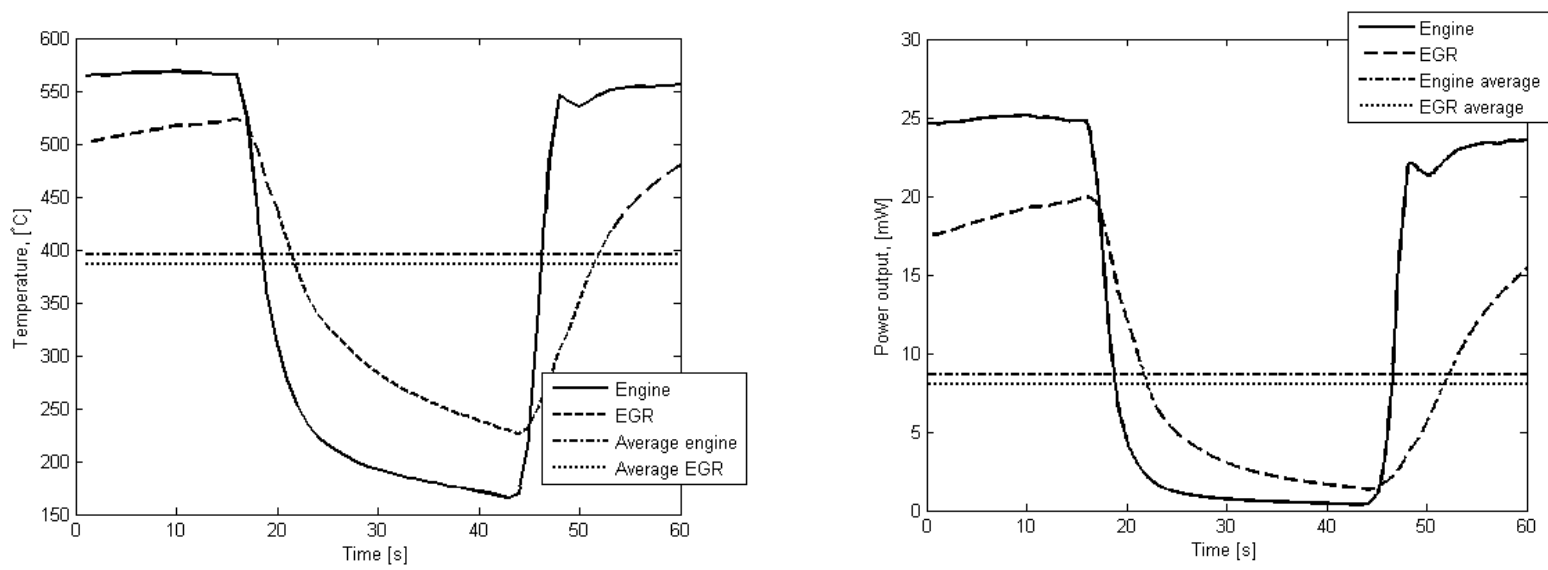

FIGURE 2. (a) Reduced gas temperature cycles (b) Power variation due to exhaust gas temperature fluctuations.

TABLE a). Temperature distribution in the TEG-EGR system.

\begin{tabular}{lcc}
\hline Domain & $\Delta \boldsymbol{T}\left[{ }^{\circ} \mathbf{C}\right]$ & $\Delta \boldsymbol{T} / \Delta \boldsymbol{T}_{\text {tot }}[\mathbf{\%}]$ \\
\hline Gas phase & 156 & 33.98 \\
Cupper & 0.137 & 0.03 \\
TE material & 303 & 65.99 \\
\hline
\end{tabular}

Since a significant amount of heat must be removed in the EGR-cooler there will be a large temperature gradient also from the inlet to the outlet of the system. Results are not included in this paper due to lack of space. However this was pointed out in a study of TEG application in engine exhaust gas systems [1] and will also occur in TEGEGR systems. The problem can be solved by placing materials with optimal performance at high temperatures close to the inlet and different materials more suitable to intermediate and lower temperature operation in the middle and end sections of the TEG-EGR respectively. The model developed in this work allows heat flow and temperature distribution inside the EGR-cooler to be quantified and allows selection of different materials to obtain an overall optimal TEG performance.

Also varying engine load will complicate the design, since the exhaust gas temperature cycles in a range approximately $130-590{ }^{\circ} \mathrm{C}$. In this study temperature fluctuation from a reduced vehicle cycle was used to analyze the effect on the performance. The simulations predict the power generation as a function of time. Energy is used to measure the mean performance, defined by Eq. 3. Figure 2a shows a reduced cycle of the exhaust gas temperature for the engine and EGR. Two performance simulations for engine and EGR cycles are compared to the performance based on the average temperature for each cycle. The results are shown in Figure $2 b$, and the energies are summarized in Table b.

$$
E=\int R_{e x t} I^{2}(t) d t
$$

TABLE b). Energy comparison over a reduced vehicle cycle.

\begin{tabular}{lcc}
\hline Analysis & $\mathbf{E}[\mathbf{J}]$ & $\mathbf{E}_{\text {transient }} / \mathbf{E}_{\text {average }}[-]$ \\
\hline Engine, transient temperature & 0,78 & 1,50 \\
Engine, average temperature & 0,52 & \\
EGR, transient temperature & 0,58 & 1,21 \\
EGR, average temperature & 0,48 & \\
\hline
\end{tabular}

As seen in Table b, higher performance is achieved when transient data with temperatures fluctuations is used, $21 \%$ and $50 \%$ higher compared to the average temperatures. Obviously these results depends on the TE material used. In this case for $\mathrm{Ba}(8) \mathrm{Ga}(16) \mathrm{Ge}(30)$ clathrates, $Z T$ (figure of merit) for the p-material increases with temperature, and for n-material $Z T_{\text {opt }}$ occurs at $500^{\circ} \mathrm{C}$. It can also be concluded From Figure $2 \mathrm{~b}$ that the contribution 
to the overall performance during the cold exhaust gas period (time when $T<T_{\text {avg }}$ in the transient data) contributes with less than $5 \%$ (engine) and $16 \%$ (EGR). Smoothing out the temperature peaks, ultimately to the average temperature of the transient data, could be successful if a material with the same temperature optimum can be used. In reality such a system is difficult to envisage since it will be associated with a weight penalty that could be difficult to motivate in vehicle applications. But it would be possible to smoothen out the temperature fluctuations to some extent. The final selection of material must therefore not only consider the temperature optimum for the $\mathrm{n}$ - and p-material but also material stability at the peak temperatures that will occur, these might be reduced from the engine peaks though.

Further these simulation results depend on the size of the elements. An approximation of the time scale for thermal diffusion through the TE material is given by $\tau=\delta^{2} / D_{H}$ (here $\delta$ is the characteristic length and $D_{H}$ is the thermal diffusivity). In these simulations the size of the thermoelectric elements is small and the time scale is approximately 3 seconds. If the size of the legs in the thermoelectric material is increased from $1.6 \mathrm{~mm}$ to $4.0 \mathrm{~mm}$ due to some reason e.g. structural integrity, the time scale increases approximately to 18 seconds. Hence a change in the size of the thermoelectric elements affect the results in the transient analysis. Nevertheless, the geometry of the TE material is specified in the 3D CFD model so the effect is automatically accounted for when simulating different TEG-EGR designs.

\section{DISCUSSION AND CONCLUSIONS}

A model that allows fluid flow, heat transfer and thermoelectric power generation in TEG-EGR systems was successfully developed. It allows critical design parameters in the systems to be identified, and allows new designs to be explored by means of virtual design. This work shows how advanced simulations methods can be used to obtain the insight to coupled phenomenon and quantify flow, heat transfer and power generation. Further it allows detailed analysis of system effects due to varying engine load and exhaust gas temperature fluctuations, and specification of design requirements on the system for integration in the vehicles.

For a TEG-EGR application the main challenge is to obtain high heat transfer and maintain a low pressure drop in the system as shown in the results section. Hence, when developing new TEG-EGR systems it is equally important to focus on the heat transfer as improving the performance of thermoelectric material. It is concluded that a new design of the heat exchanger is required to achieve an efficient system with low pressure drop. Further simulations using transient data of the exhaust gas is recommended, to determine whether the optimum performance occurs when smoothing out the peaks or when running the system with high peak temperatures. The answer depends on over what temperature range the TE material can maintain a high performance and at the same time be stable for the high temperature peaks.

\section{ACKNOWLEDGMENTS}

This work was supported by The Swedish Foundation for Strategic Environmental Research, through the research program E4-MISTRA. The goal with E4-MISTRA is to reduce vehicle emissions of nitrogen oxides and particulates, without increasing energy consumption and hence releases of carbon dioxide. The authors would like to thank Volvo Technology AB for supporting the transient engine and EGR exhaust gas data.

\section{REFERENCES}

[1] N. Espinosa, M. Lazard, L. Aixala,H. Scherrer. Modeling a Thermoelectric Generator Applied to Diesel Automotive Heat Recovery. Journal of Electronic Materials. 39(9) (2010), 1446-1455.

[2] B. Andersson, R. Andersson, L. Håkansson, M. Mortensen, R. Sudiyo,B.G.M. van Wachem, Computational Fluid Dynamics for Engineers. 2011, Cambridge: Cambridge University Press.

[3] M. Chen, L.A. Rosendahl,T. Condra. A three-dimensional numerical model of thermoelectric generators in fluid power systems. International Journal of Heat and Mass Transfer. 54(1-3) (2011), 345-355.

[4] D. Cederkrantz, A. Saramat, G.J. Snyder,A.E.C. Palmqvist. Thermal stability and thermoelectric properties of p-type $\mathrm{Ba}(8) \mathrm{Ga}(16) \mathrm{Ge}(30)$ clathrates. Journal of Applied Physics. 106(7) (2009). 